\title{
SOME REMARKS ON THE CLASS OF CONTINUOUS (SEMI-) STRICTLY QUASICONVEX FUNCTIONS
}

\author{
ARIS DANIILIDIS \& YBOON GARCIA RAMOS
}

\begin{abstract}
We introduce the notion of variational (semi-) strict quasimonotonicity for a multivalued operator $T: X \rightrightarrows X^{*}$ relative to a nonempty subset $A$ of $X$ which is not necessarily included in the domain of $T$. We use this notion to characterize the subdifferentials of continuous (semi-) strictly quasiconvex functions. The proposed definition is a relaxation of the standard definition of (semi-) strict quasimonotonicity, the latter being appropriate only for operators with nonempty values. Thus, the derived results are extensions to the continuous case of corresponding results for locally Lipschitz functions.
\end{abstract}

\section{INTRODUCTION}

The notion of quasiconvexity, referring to a function whose sublevel sets are convex, is probably the most natural generalization of convexity. Its simple definition makes it the starting point for further investigations (see [22], [4], [20], [9], for example).

Comparing with the convex case, a quasiconvex function might have local minima which are not global (typical failure in almost all deterministic global optimization methods), or critical points which are not local minimizers (typical failure in all Newton type minimization methods). In this work we are interested in the important subclasses of semistrictly (respectively, strictly) quasiconvex functions, for which at least the first inconvenience is overcome. These classes meet a large domain of applications in microeconomics (see [4] and references therein) and recently regained attention due to its applications to the multicriteria (Pareto) optimization problem (see recent developments in [5], [6]).

After the developments in the area of non-smooth analysis [8], there has been an effort to characterize the generalized convexity of functions in terms

2000 Mathematics Subject Classification. Primary 26B42 ; Secondary 49J52, 90C26, 91B42.

Key words and phrases. Quasiconvex function, quasimonotone operator, variational analysis, utility function. 
of generalized monotonicity of their subdifferential (see for instance [19], [21], [1], [10]). As an outcome, a natural correspondence between notions of generalized convexity and of generalized monotonicity arises, reflecting the known duality between utility functions (typically being semistrictly quasiconvex) and demand correspondences (typically being semistrictly quasimonotone multifunctions) in the consumer's theory (see [4], [14], for example).

The aforementioned correspondence has been progressively extended from the differentiable case to the case of continuous or sometimes even lower semicontinuous functions. The main tool for these extensions is a nonsmooth version of the mean value theorem, which is known to be valid for every reasonable choice of subdifferential (see [2] for details). However, besides the importance of the classes of semistrict (respectively, strict) quasiconvexity, their dual characterization in terms of the semistrict (respectively, strict) quasimonotonicity of their subdifferentials cannot be extended beyond the class of locally Lipschitz functions, due to the restrictions the aforementioned definitions impose on the domain of the operator.

In this work we propose a variational modification of the definitions of semistrict (respectively, strict) quasimonotonicity to make them operational to the continuous case. These definitions enter naturally to the aforementioned duality scheme, and entail no change in case of subdifferentials of locally Lipschitz functions.

The paper is organized as follows: in Section 2 we fix our notation and recall definitions and basic results. In Section 3 we introduce the variational definitions of the semistrict and the strict quasimonotonicity (Definition 2), we use them to establish the subdifferential characterizations of the semistrict quasiconvexity (Theorem 5) and the strict quasiconvexity (Theorem 7). In the last part we discuss the compatibility of the introduced notions for operators that are subdifferentials of locally Lipschitz functions.

\section{NOTATION AND BASIC DEFINITIONS}

Throughout this work $X$ will denote a Banach space, $X^{*}$ its topological dual and $\left\langle x^{*}, x\right\rangle$ the value of the functional $x^{*} \in X^{*}$ at $x \in X$. For any $x, y \in X$ we set

$$
[x, y]:=\{t x+(1-t) y: 0 \leq t \leq 1\}
$$

and define the segments $] x, y],[x, y[$ and $] x, y[$ analogously. We denote by $B(x, r)$ the open ball of center $x \in X$ and radius $r>0$ and for any nonempty subset $S$ of $X$ we denote by co $S$ its convex envelope. 
Given a lower semicontinuous function $f: X \rightarrow \mathbb{R} \cup\{+\infty\}$ we denote by

$$
\operatorname{dom} f:=\{x \in X: f(x)<+\infty\}
$$

its domain (which we always assume nonempty) and given $x \in \operatorname{dom} f$ we denote by

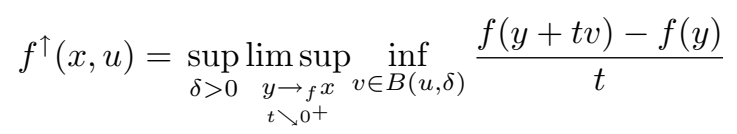

its Clarke-Rockafellar generalized derivative, where $t \searrow 0^{+}$indicates the fact that $t>0$ and $t \rightarrow 0$, and $y \rightarrow_{f} x$ means that both $y \rightarrow x$ and $f(y) \rightarrow f(x)$ (this is superfluous, if $f$ is assumed continuous). Then the Clarke-Rockafellar subdifferential $\partial f(x)$ of the function $f$ at the point $x$ is defined as follows $([8])$ :

$$
\partial f(x)=\left\{x^{*} \in X: f^{\uparrow}(x, u) \geq\left\langle x^{*}, u\right\rangle, \quad \text { for all } u \in X\right\} .
$$

Let us recall (see [4] or [9] for example) that the function $f$ is called:

- quasiconvex, if for all $x, y \in \operatorname{dom}(f)$

$$
f(z) \leq \max \{f(x), f(y)\}, \forall z \in[x, y]
$$

- semistrictly quasiconvex, if for all $x, y \in \operatorname{dom}(f)$ the following implication holds:

$$
f(x)<f(y) \Rightarrow f(z)<f(y), \forall z \in] x, y[;
$$

- strictly quasiconvex, if for all $x, y \in \operatorname{dom}(f)$

$$
f(z)<\max \{f(x), f(y)\}, \forall z \in] x, y[.
$$

Let further $T: X \rightrightarrows X^{*}$ denote a multivalued operator with domain

$$
\operatorname{dom}(T):=\{x \in X: T(x) \neq \emptyset\}
$$

and graph

$$
\operatorname{Gr}(T):=\left\{\left(x, x^{*}\right) \in X \times X^{*}: x^{*} \in T(x)\right\} .
$$

Let $A$ be a nonempty subset of $X$. The operator $T$ is called:

- quasimonotone on $A$, if for all $x_{1}, x_{2} \in A$, all $x_{1}^{*} \in T\left(x_{1}\right)$ and all $x_{2}^{*} \in T\left(x_{2}\right)$ the following implication holds:

$$
\left\langle x_{1}^{*}, x_{2}-x_{1}\right\rangle>0 \Rightarrow\left\langle x_{2}^{*}, x_{2}-x_{1}\right\rangle \geq 0 .
$$

- semistrictly quasimonotone on $A$, if it is quasimonotone on $A$ and for all $x_{1}, x_{2} \in A$ and $x_{1}^{*} \in T\left(x_{1}\right)$ we have

$$
\left.\left\langle x_{1}^{*}, x_{2}-x_{1}\right\rangle>0 \Longrightarrow \exists z \in\right] \frac{x_{1}+x_{2}}{2}, x_{2}\left[, \exists z^{*} \in T(z):\left\langle z^{*}, x_{2}-x_{1}\right\rangle>0\right. \text {. }
$$


- strictly quasimonotone on $A$, if it is quasimonotone on $A$ and for every $x_{1}, x_{2} \in A$ we have

$$
\left.x_{1} \neq x_{2} \Longrightarrow \exists z \in\right] x_{1}, x_{2}\left[, \exists z^{*} \in T(z):\left\langle z^{*}, x_{2}-x_{1}\right\rangle \neq 0 .\right.
$$

The definition of quasimonotonicity is classical (see [17], [4], [12], [9], for example) and is associated with the following result, which is a cornerstone of quasiconvex analysis (see [19], [21], [1] or [11, Theorem 2.1], for example).

Theorem 1. Let $f: X \longrightarrow \mathbb{R} \cup\{+\infty\}$ be a lower semicontinuous function. The following assertions are equivalent:

(a) $f$ is quasiconvex ;

(b) $\partial f$ is quasimonotone;

(c) for all $x, y \in \operatorname{dom}(f)$ :

$\exists x^{*} \in \partial f(x):\left\langle x^{*}, y-x\right\rangle>0 \Rightarrow f(z) \leq f(y)$, for all $z \in[x, y]$.

The notions of semistrict/strict quasimonotonicity were first introduced in [18] as extensions of corresponding notions for functions (that is, singlevalued operators) considered in [16]. In this latter work ([16]) it is established that a differentiable semistrictly (respectively, strictly) quasiconvex functions is characterized by the semistrict (respectively, strict) quasimonotonicity of its derivatives. In [11] the aforementioned result is extended to the class of locally Lipschitz functions $f$, where (4) (respectively, (5)) is used to define the semistrict (respectively, strict) quasimonotonicity for the (multivalued) Clarke subdifferential $\partial f$. The proof, heavily based on the Lebourg Mean Value theorem (see [8], for example), leaves no hope for further extensions: indeed, in contrast to the classical definitions of monotonicity or quasimonotonicity, the considered definitions yield implicitly the nonemptiness of the operator $T$ on a dense subset of every segment $\left[x_{1}, x_{2}\right]$. Concurrently, outside the class of locally Lipschitz functions, the domain of the Clarke-Rockafellar subdifferential $\partial f$ can easily be empty on whole lines.

To establish a duality between semistrict quasiconvexity and semistrict quasimonotonicity beyond the Lipschitzian case, a different approach is adapted in [3]. That approach, inspired from a previous work of Borde and Crouzeix [7], consisted in replacing the subdifferential $\partial f$ of $f$ by the operator "normal cone to the sublevel sets" (denoted by $N_{f}$ ). In doing so, an application of the Hahn-Banach theorem recompenses the need of using an approximate Mean Value Theorem. As a result, the aforementioned duality is extended to the class of continuous functions in this new framework.

The aim of this work is to show that an analogous duality scheme based on the notion of subdifferential is plausible, provided a variational adaptation of the notions defined by (4) and (5) to make them compatible with 
the approximate Mean Value theorem. In the last part of this work (cf. Corollary 8 and Proposition 9) we show how these new definitions relate to other notions of generalized monotonicity.

\section{MAin Result}

The following notation will be used in the sequel. For every $x_{1}, x_{2} \in X$ we set

$$
D_{T}\left(x_{1}, x_{2}\right):=
$$

$=\{z \in] x_{1}, x_{2}\left[: \exists\left(z_{n}, z_{n}^{*}\right)_{n \geq 1} \subset \operatorname{Gr}(T), \lim _{n} z_{n}=z \&\left\langle z_{n}^{*}, x_{2}-z_{n}\right\rangle>0\right\}$.

The following notions will play a central role in this work.

Definition 2. Let $A$ be a nonempty convex subset of $X$. An operator $T: X \rightrightarrows X^{*}$ is called

(i) variationally semistrictly quasimonotone on $A$, if it is quasimonotone on $A$ and for every $x_{1}, x_{2} \in A$ and $x_{1}^{*} \in T\left(x_{1}\right)$ we have

$$
\left.\left.\left\langle x_{1}^{*}, x_{2}-x_{1}\right\rangle>0 \Longrightarrow D_{T}\left(x_{1}, x_{2}\right) \cap\right] \frac{x_{1}+x_{2}}{2}, x_{2}\right] \neq \emptyset ;
$$

(ii) variationally strictly quasimonotone on $A$, if it is quasimonotone on $A$ and for every $x_{1}, x_{2} \in A$ there exist $\left.z \in\right] x_{1}, x_{2}[$ and $\delta>0$ such that for some $i_{0} \in\{1,2\}$ and all $z^{\prime} \in B(z, \delta)$ we have

$$
D_{T}\left(z^{\prime}, x_{i_{0}}\right) \neq \emptyset \text {. }
$$

Remark 3. (i) Assuming (4) and taking $z_{n}:=z$ for all $n \geq 1$ we see that (7) holds. Thus every semistrictly quasimonotone operator is variationally semistrictly quasimonotone. On the other hand, properties (5) and (8) are not directly comparable. Nevertheless, as we show in Corollary 8, strict (respectively, semistrict) quasimonotonicity and variational strict (respectively, semistrict) quasimonotonicity coincide for subdifferentials of locally Lipschitz functions.

(ii) Variational strict quasimonotonicity implies variational semistrict quasimonotonicity. Indeed, let us assume $\left\langle x_{1}^{*}, x_{2}-x_{1}\right\rangle>0$ for some $x_{1}, x_{2} \in A$ and $x_{1}^{*} \in T\left(x_{1}\right)$. Setting $w=\frac{x_{1}+x_{2}}{2}$ and applying (8) on the segment $\left[w, x_{2}\right]$ we deduce that for some $z \in] w, x_{2}\left[\right.$ and $\delta>0$ and for all $z^{\prime} \in B(z, \delta)$ we either have $D_{T}\left(z^{\prime}, x_{2}\right) \neq \emptyset$ (thus in particular, $D_{T}\left(z, x_{2}\right) \neq \emptyset$, yielding $\left.\left.D_{T}\left(x_{1}, x_{2}\right) \cap\right] w, x_{2}\right] \neq \emptyset$ and we are done) or $D_{T}\left(z^{\prime}, w\right) \neq \emptyset$. In the second case we obtain a sequence $\left\{z_{n}\right\} \longrightarrow z$ with $z_{n}^{*} \in T\left(z_{n}\right)$ and $\left\langle z_{n}^{*}, w-z_{n}\right\rangle>0$. On the other hand, by quasimonotonicity we infer that $\left\langle z_{n}^{*}, z_{n}-x_{1}\right\rangle \geq 0$. Multiplying the first inequality by 2 and adding to the second one we obtain $\left\langle z_{n}^{*}, x_{2}-z_{n}\right\rangle>0$. Thus $D_{T}\left(w, x_{2}\right) \neq \emptyset$, which yields $(7)$. 
We now show that the above variational definitions characterize the subdifferentials of the continuous semistrictly (respectively, strictly) quasiconvex functions. We shall need the following result concerning the class of continuous quasiconvex functions (see [4]). We include a short proof for the reader's convenience.

Proposition 4. Let $f: X \rightarrow \mathbb{R} \cup\{+\infty\}$ be a continuous quasiconvex function. Then $f$ is semistrictly quasiconvex if, and only if, the set of local and global minimizers of $f$ coincide.

Proof For the needs of this proof we denote by L-min $f$ (respectively, $\mathrm{G}-\min f$ ) the set of local (respectively, global) minimizers of $f$.

Let us first assume $f$ is semistrictly quasiconvex and let us show that $\mathrm{L}-\min f=\mathrm{G}$ - $\min f$ (continuity is not needed for this implication). Let us assume, towards a contradiction, that there exists $y \in \mathrm{L}-\min f$ and $x \in X$ with $f(y)>f(x)$. Then by quasiconvexity, the restriction of $f$ to the segment $[x, y]$ should be increasing near $y$. Since $y$ is local minimum, this implies $f$ is constant on $[z, y]$ for some $z \in] x, y[$, which clearly contradicts (2).

Let us now assume $f$ is quasiconvex and continuous and L-min $f=$ $\mathrm{G}-\min f$. If $f$ were not semistrictly quasiconvex, then we would have $f(x)<f(z)=f(y)$, for some $x, y \in \operatorname{dom} f$ and $z \in] x, y[$. Since $y$ cannot be a local minimum of $f$, there exists $\left(y_{n}\right) \rightarrow y$ with $f\left(y_{n}\right)<f(y)$. Let $t=\|x-y\|^{-1}\|z-y\|$ and set $x_{n}=y_{n}+\frac{1}{t}\left(z-y_{n}\right)$. Using the quasiconvexity of $f$, we deduce from the relation $f(z)=f(y)>f\left(y_{n}\right)$ that $f\left(x_{n}\right) \geq f(y)$. Since $\left(x_{n}\right) \rightarrow x$ and $f(x)<f(y)$ we obtain a contradiction to the continuity of $f$ at $x$.

The following result relates variational semistrict quasimonotonicity to semistrict convexity in the continuous case.

Theorem 5. Let $f: X \rightarrow \mathbb{R} \cup\{+\infty\}$ be a continuous function with a convex domain $\operatorname{dom} f$.

The following assertions are equivalent:

(i) $f$ is semistrictly quasiconvex;

(ii) for all $x, y \in \operatorname{dom} f$ and $x^{*} \in \partial f(x)$ we have

$$
\left\langle x^{*}, y-x\right\rangle>0 \Rightarrow f(z)<f(y), \quad \text { for all } z \in[x, y[;
$$

(iii) $\partial f$ is variationally semistrictly quasimonotone on $\operatorname{dom} f$.

Proof Throughout the proof we set $K=\operatorname{dom} f$ and $T=\partial f$.

(i) $\Longrightarrow$ (ii). Let $x, y \in K$ and $x^{*} \in \partial f(x)$ with $\left\langle x^{*}, y-x\right\rangle>0$. In view of (2), it is sufficient to show that $f(x)<f(y)$. Assuming this is not the 
case, then by quasiconvexity, $f$ has to be constant on the segment $[x, y]$ (cf. Theorem 1(c)). Applying [11, Lemma 2.1] we obtain that every $z \in] x, y]$ is a local minimum of $f$ while $x$ is not a local minimum. Since $f(x)=f(z)$, we obtain a contradiction to Proposition 4.

(ii) $\Longrightarrow$ (iii). Let us assume (ii) holds. Evoking again Theorem $1[(\mathrm{c}) \rightarrow(\mathrm{b})]$ we obtain that $\partial f$ is quasimonotone. Let us now assume $x, y \in K$ and $x^{*} \in \partial f(x)$ be such that $\left\langle x^{*}, y-x\right\rangle>0$. Then $f(z)<f(y)$, for all $z \in[x, y[$. Taking $z$ near $y$ such that $\|z-y\|<\frac{1}{2}\|x-y\|$, and applying the approximate mean value theorem on the segment $[z, y]$ (see [2, Corollary 4.3], for example) we obtain directly that $D_{T}(z, y) \neq \emptyset$, thus $D_{T}(x, y) \neq \emptyset$. This shows that $\partial f$ is variationally semistrictly quasimonotone on $K$.

(iii) $\Longrightarrow($ i). Let us assume (iii) holds. Then $\partial f$ is in particular quasimonotone, thus $f$ is quasiconvex. Let us assume, towards a contradiction, that $f$ is not semistrictly quasimonotone. Then by Proposition 4, there exist $x, y \in K$ with $f(x)<f(y)$ and $y$ is a local minimum of $f$. Using this information and the continuity of $f$, we deduce that there exists $\delta, \delta_{1}>0$ such that

$$
f\left(y^{\prime}\right) \geq f(y)>f\left(x^{\prime}\right), \quad \text { for all } x^{\prime} \in B(x, \delta) \text { and } y^{\prime} \in B\left(y, \delta_{1}\right) .
$$

Set $C=\operatorname{co}(B(x, \delta) \cup\{y\}) \backslash\{y\}$. Then (9) together with the quasiconvexity of $f$ yield that $f$ is constant on $C \cap B\left(y, \delta_{1}\right)$. Since the latter set is open, we deduce

$$
\partial f\left(y^{\prime}\right)=\{0\}, \quad \text { for all } y^{\prime} \in C \cap B\left(y, \delta_{1}\right) .
$$

We may also assume $\delta_{1}>0$ to be maximal with respect to the above property. Taking now any $x_{1} \in C \backslash B\left(y, \delta_{1}\right)$ such that $\left\|x_{1}-y\right\|<2 \delta_{1}$ we have $f\left(x_{1}\right)<f(y)$. Applying the approximate mean value theorem $([2$, Corollary 4.3]) to the segment $\left[x_{1}, y\right]$ we obtain a sequence $\left(z_{n}\right) \longrightarrow z \in$ $\left[x_{1}, y\left[\right.\right.$ with $\left\langle z_{n}^{*}, y-z_{n}\right\rangle>0$. Taking $n$ sufficiently large, we ensure that $z_{n} \in C$ and $\left\|z_{n}-y\right\|<2 \delta_{1}$. Applying now the definition of semistrict quasimonotonicity (cf. relation (7)) to the segment $\left[z_{n}, y\right]$, we infer that

$$
D_{T}\left(z_{n}, y\right) \cap B\left(y, \delta_{1}\right) \neq \emptyset .
$$

This clearly contradicts (10).

Remark 6. As already mentioned, replacing variational semistrict quasimonotonicity by semistrict quasimonotonicity (cf. relation $((4))$ in the statement of Theorem 5, does not lead to a full equivalence in the continuous case. The best partial result in this direction was recently established in [15, Theorem 7] (namely, (iii) $\Longrightarrow($ ii) $\Longleftrightarrow(i)$ ).

We are now ready to state the second main result of this work, relating variational strict quasimonotonicity to strict convexity. 
Theorem 7. Let $f: X \rightarrow \mathbb{R} \cup\{+\infty\}$ be a continuous function with a convex domain $\operatorname{dom} f$.

The following assertions are equivalent:

(i) $f$ is strictly quasiconvex;

(ii) $\partial f$ is variationally strictly quasimonotone on $\operatorname{dom} f$.

Proof We set again $K=\operatorname{dom} f$ and $T=\partial f$.

(i) $\Longrightarrow$ (ii). Since by Theorem 1 (b) we have that $\partial f$ is quasimonotone, we need only show that (8) holds. To this end, let $x_{1}, x_{2} \in K$ and pick any $z \in] x_{1}, x_{2}\left[\right.$. Using (3) we deduce that $f(z)<f\left(x_{i_{0}}\right)$ for some $i_{0} \in\{1,2\}$. Since $f$ is continuous, there exists $\delta>0$ such that for all $z^{\prime} \in B(z, \delta)$ we have $f\left(z^{\prime}\right)<f\left(x_{i_{0}}\right)$. Applying the approximate mean value theorem ([2, Corollary 4.3]) on each segment $\left[z^{\prime}, x_{i_{0}}\right]$ we obtain directly that $D_{T}\left(z^{\prime}, x_{i_{0}}\right) \neq$ $\emptyset$.

(ii) $\Longrightarrow($ i). Assume $\partial f$ is variationally strictly quasimonotone on $\operatorname{dom} f$. Then by Remark 3 and Theorem $5[($ iii $) \rightarrow(\mathrm{i})]$ we deduce that $f$ is semistrictly quasiconvex. Let us assume, towards a contradiction, that $f$ is constant in a non-trivial segment $\left[x_{1}, x_{2}\right]$ of $K$ taking the value $a \in \mathbb{R}$ there. We deduce from (8) that there exist $i_{0} \in\{1,2\}$ and $\left(u, u^{*}\right) \in \operatorname{Gr} \partial f$ such that $\left\langle u^{*}, x_{i_{0}}-u\right\rangle>0$. In view of Theorem 5 (ii) this yields

$$
a=f\left(x_{i_{0}}\right)>f(u) \geq \inf f .
$$

Thus $f$ does not attain its global minimum on the segment $\left[x_{1}, x_{2}\right]$. Let us denote by

$$
S_{f}(a):=\{x \in X: f(x) \leq a\}
$$

the (closed convex) sublevel set of $f$. The above inequality together with Proposition 4 show that the interior of $S_{f}(a)$ is nonempty and coincides with the strict sublevel set $S_{f}^{-}(a)=\{x \in X: f(x)<a\}$. It follows that the segment $\left[x_{1}, x_{2}\right]$ is in the boundary of the open convex set $S_{f}^{-}(a)$ and $\left[x_{1}, x_{2}\right] \cap S_{f}^{-}(a)=\emptyset$. By the Hahn-Banach separation theorem we obtain a linear functional $p \in X^{*}$ and $\beta \in \mathbb{R}$ such that $\langle p, z\rangle=\beta>\langle p, y\rangle$ for all $z \in\left[x_{1}, x_{2}\right]$ and $y \in S_{f}^{-}(a)$. Set $U=\{x \in X:\langle p, x\rangle>\beta\}$. Then $U$ is an open subset of $X \backslash S_{f}(a)$ containing the segment $\left[x_{1}, x_{2}\right]$ in its closure. Let now $z \in] x_{1}, x_{2}\left[, \delta>0\right.$ and $i_{0} \in\{1,2\}$ be given by (8) applied to the segment $\left[x_{1}, x_{2}\right]$ and let us define

$$
C=\operatorname{co}\left[(B(z, \delta) \cap U) \cup\left\{x_{i_{0}}\right\}\right] .
$$

For any $z^{\prime} \in B(z, \delta) \cap U$, we infer from (8) that $D_{T}\left(z^{\prime}, x_{i_{0}}\right) \neq \emptyset$, which in view of (7) yields the existence of some $u \in C \backslash\left\{x_{i_{0}}\right\}$ and $u^{*} \in \partial f(u)$ satisfying $\left\langle u^{*}, x_{i_{0}}-u\right\rangle>0$. It follows from Theorem5(ii) that $a=$ 
$f\left(x_{i_{0}}\right)>f(u)$. On the other hand, it is easily seen that $C \backslash\left\{x_{i_{0}}\right\} \subset U$, thus $f(u)>a$, which is a contradiction.

A direct consequence of the above characterizations is the following result.

Corollary 8. Let $T=\partial f$ for some locally Lipschitz function $f$ (defined on a nonempty convex subset $A$ of $X)$. Then

(i) $T$ is semistrictly quasimonotone (on the set $A$ ) if and only if it is variationally semistrictly quasimonotone (on the set $A$ ).;

(ii) $T$ is strictly quasimonotone (on the set $A$ ) if and only if it is variationally strictly quasimonotone (on the set $A$ ).

Proof (i) It follows from the observation that both notions of semistrict quasimonotonicity and of variational semistrict quasimonotonicity characterize the semistrict quasiconvexity of $f$ in the locally Lipschitz case (cf. Theorem 5 and [11, Theorem 3.2]).

(ii) Similarly, if $f$ is locally Lipschitz, then both notions of strict and of variational strict quasimonotonicity characterize the strict quasiconvexity of the function (cf. Theorem 7 and [11, Theorem 4.1]).

Let us finally see how the variational semistrict quasimonotonicity relates to the concept of proper quasimonotonicity. We recall from [10, Definition 4.2] that an operator $T: X \rightrightarrows X^{*}$ is called

- properly quasimonotone on a convex subset $A$ of $X$, if for every $\left\{x_{1}, x_{2}, \ldots, x_{k}\right\} \subset A$ and every $y \in \operatorname{co}\left\{x_{j}\right\}_{j \in \mathbb{N}_{k}}$, there exists $i \in \mathbb{N}_{k}$ such that

$$
\forall x_{i}^{*} \in T\left(x_{i}\right),\left\langle x_{i}^{*}, y-x_{i}\right\rangle \leq 0 .
$$

It is sufficient to take $k=2$ and $y=\frac{x_{1}+x_{2}}{2}$ to see that every properly quasimonotone operator is quasimonotone. The converse implication (being in general false) is true if either $X=\mathbb{R}$ or $T=\partial f$ for some continuous function $f$ which has to be quasiconvex (see [10] for details). In [11, Proposition 5.1] it is shown that any semistrictly quasimonotone operator is properly quasimonotone. Let us now show that the same happens for any variationally semistrictly quasimonotone operator.

Proposition 9. Let $A$ be a nonempty convex subset of $X$ and $T: X \rightrightarrows X^{*}$. If $T$ is variationally semistrictly quasimonotone on $A$ then it is also properly quasimonotone on $A$.

Proof Let as assume that $T$ is variationally semistrictly quasimonotone on $A$ and not properly quasimonotone there. Then there exist $\left\{x_{1}, x_{2}, \ldots x_{k}\right\} \subset$ 
$A$ and $y \in \operatorname{co}\left\{x_{j}\right\}_{j \in \mathbb{N}_{k}}$ such that for every $i \in \mathbb{N}_{k}$ there exists $x_{i}^{*} \in T\left(x_{i}\right)$ satisfying

$$
\left\langle x_{i}^{*}, y-x_{i}\right\rangle>0 .
$$

By continuity, the strict inequality above remains true if we replace $y$ by any $w \in B(y, \delta)$ (for $\delta>0$ sufficiently small). Thus, by quasimonotonicity of $T$ we obtain $\left\langle w^{*}, w-x_{i}\right\rangle \geq 0$, for all $w^{*} \in T(w)$. Since this is true for all $i \in \mathbb{N}_{k}$ we conclude that for all $w \in B(y, \delta)$ and all $w^{*} \in T(w)$

$$
\left\langle w^{*}, w-y\right\rangle \geq 0 .
$$

We may assume with no loss of generality that $r:=\left\|y-x_{1}\right\| \leq\left\|y-x_{i}\right\|$, for all $i \in \mathbb{N}_{k}$. Since $\left\langle x_{1}^{*}, y-x_{1}\right\rangle>0$ we have by Definition 2 that $D_{T}\left(x_{1}, y\right) \cap$ $B\left(y, \frac{r}{2}\right) \neq \emptyset$. This yields the existence of $z \in B\left(y, \frac{r}{2}\right)$ and $z^{*} \in T(z)$ such that $\left\langle z^{*}, y-z\right\rangle>0$. Repeating the same argument (on the segment $[z, y]$ ) we see that we can get arbitrarily close to $y$. In particular there exist $\bar{w} \in B(y, \delta)$ and $\bar{w}^{*} \in T(\bar{w})$ such that $\left\langle\bar{w}^{*}, y-\bar{w}\right\rangle>0$. This clearly contradicts (12).

We recall (see [13], for example) that a point $x_{0} \in A$ is said to be a solution for the Minty Variational Inequality problem of the operator $T: X \rightrightarrows X^{*}$ with respect to the (nonempty) subset $A$ of $X$, provided that for every $\left(y, y^{*}\right) \in \operatorname{Gr} T$ with $y \in A$ we have $\left\langle y^{*}, y-x\right\rangle \geq 0$. Combining Proposition 9 with [11, Theorem 5.1] (see also [13]) we obtain the following

Corollary 10. If $T$ is variationally semistrictly quasimonotone, then the Minty variational inequality has nonempty solutions on every nonempty convex and weakly compact subset $A$ of $X$.

Acknowledgement Part of this work was accomplished during a research visit of the first author to the University of Antilles-Guyane (February 2005) and of the second author to the CRM of the Autonomous University of Barcelona (July 2005). The authors wish to thank their hosts for the hospitality.

\section{REFERENCES}

[1] Aussel, D., Subdifferential Properties of Quasiconvex and Pseudoconvex Functions: A Unified Approach, J. Optim. Theory Appl. 97, (1998) 29-45.

[2] Aussel, D., Corvellec, J.-N., \& Lassonde, M., Mean Value Property and Subdifferential Criteria for Lower Semicontinuous Functions, Trans. Amer. Math. Soc. 347 (1995), 4147-4161.

[3] Aussel, D., \& Danillidis, A., Normal characterization of the main classes of quasiconvex functions, Set-Valued Anal. 8 (2000), 219-236.

[4] Avriel, M., Diewert, W.-E., Schaible, S. \& Zang, I., Generalized Concavity, (Plenum Publishing Corporation, New York, New York, 1988). 
[5] Benoist, J., Connectedness of the Efficient Set for Strictly Quasiconcave Sets, J. Optim. Theory Appl. 96 (1998), 627-654.

[6] Benoist, J. \& Popovici, N., Contractibility of the efficient frontier of three dimensional simply-shaded sets, J. Optim. Theory Appl. 111 (2001), 81-116.

[7] Borde, J. \& Crouzeix, J.-P., Continuity Properties of the Normal Cone to the Level Sets of a Quasiconvex Function, J. Optim. Theory Appl. 66 (1990), 415-429.

[8] Clarke, F.H., Optimization and Nonsmooth Analysis, Wiley Interscience, New York, New York (1983).

[9] Crouzeix, J.-P., Generalized convexity and generalized monotonicity, Monografias del Instituto de Matematica y Ciencias Afines, IMCA, Lima, (2000).

[10] Danillidis, A. \& Hadjisavvas, N., On the subdifferentials of quasiconvex and pseudoconvex functions and cyclic monotonicity, J. Math. Anal. Appl. 237 (1999), 30-42.

[11] Danillidis, A., \& Hadjisavvas, N., Characterization of nonsmooth semistrictly quasiconvex and strictly quasiconvex functions, J. Optim. Theory Appl. 102 (1999), 525-536.

[12] Ellaia, R. \& Hassouni, A., Characterization of nonsmooth functions through their generalized gradient, Optimization 22 (1991), 401-416.

[13] John, R., A note on Minty variational inequalities and generalized monotonicity, Generalized convexity and generalized monotonicity (Karlovassi 1999), 240-246, Lecture Notes in Econom. and Math. Systems 502, Springer, Berlin (2001).

[14] John, R., The concave nontransitive consumer. Special issue: Applications to economics, J. Global Optim. 20 (2001), 297-308.

[15] Hadjisavvas, N., The Use of Subdifferentials for studying Generalized Convex Functions, J. Stat. Manag. Syst. 5 (2002), 125-139.

[16] Hadjisavvas, N. \& Schaible, S., On strong pseudomonotonicity and (semi)strict quasimonotonicity, J. Optim. Theory Appl. 79 (1993), 139-155.

[17] Karamardian, S. \& Schaible, S., Seven Kinds of Monotone Maps, J. Optim. Theory Appl. 66 (1990), 37-46.

[18] Luc, D.-T., Generalized monotone set-valued maps and support bifunctions, Acta Math. Vietnam. 21 (1996), 213-252.

[19] Luc, D.-T., Characterisations of Quasiconvex Functions, Bull. Austr. Math. Soc. 48 (1993), 393-406.

[20] Martinez-Legaz, J. E., Quasiconvex duality theory by generalized conjugation methods, Optimization 19 (1988), 603-652.

[21] Penot, J.-P. \& Quang, P.-H., Generalized Convexity of Functions and Generalized Monotonicity of Set-Valued Maps, J. Optim. Theory Appl. 92 (1997), 343-356.

[22] Penot, J.-P. \& Volle, M., Dualité de Fenchel et Quasiconvexité, C. R. Acad. Sci. Paris, Serie I, t. 304 (1987), 371-374.

\author{
Aris DANIILIDIS \\ Departament de Matemàtiques \\ Universitat Autònoma de Barcelona \\ E-08193 Bellaterra (Cerdanyola del Vallès), Spain. \\ e-mail: arisd@mat.uab.es \\ http://mat.uab.es/ arisd
}




\section{Yboon GARCIA RAMOS}

Département de Mathématiques

Laboratoire d'analyse, optimisation et contrôle

Université des Antilles et de la Guyane

F-97159 Pointe-à-Pître (Campus de Fouillole), France

e-mail: yboon.garcia@univ-ag.fr 